\title{
Modelling of Mixed Matrix Membranes Using a Resistance Based Model
}

\author{
Arian Ebneyamini, Hoda Azimi, Jules Thibault, F. Handan Tezel \\ Department of Chemical and Biological Engineering, University of Ottawa \\ Ottawa, Ontario, Canada K1N 6N5 \\ Aebne013@uottawa.ca; Hazim087@uottawa.ca; Jules.Thibault@uottawa.ca; Handan.Tezel@uottawa.ca
}

\begin{abstract}
In this study, a model for the prediction of the mass transport through mixed matrix membranes (MMMs) for pervaporation and gas separation processes has been introduced. A Resistance-Based (RB) model was used in conjunction with a Finite Difference (FD) model to derive an analytical model for calculating the effective permeability of mixed matrix membranes. The proposed model was validated using experimental data for the pervaporation and gas separation applications using MMMs.
\end{abstract}

Keywords: Mixed Matrix Membranes, Effective permeability, Resistance-Based Model, Finite Difference Method

\section{Introduction}

The theoretical description of the mass transport through mixed matrix membranes is paramount to optimize the membrane separation processes. Various models such as Maxwell [1], Bruggeman [2], Lewis-Nielson [3], [4], te Hennepe [5] and Pal [6] have been introduced to estimate the effective permeability of ideal mixed matrix membranes. These models are based on the permeability of the continuous phase, permeability of the dispersed phase and the volume fraction of the solid fillers within the polymer matrix [7].

In this work, it was desired to resort to a simple predictive model such that a Resistance-Based (RB) model has been introduced to estimate the effective permeability of migrating components in a homogenously dispersed mixed matrix membrane with the assumption of ideal polymer-filler interface morphology. In addition, an accurate solution was obtained by Finite Differences (FD) to predict the effective permeability of the mixed matrix membranes for different ratios of the respective permeability in the two phases and different volumetric filler contents within the polymer matrix. The results of these two models were compared to modify the RB model by a correction factor to account for the threedirectional (3D) diffusional pathways. The new model was validated with different sets of experimental data for pervaporation and gas separation processes obtained from previously published studies.

\section{RB Model}

A resistance-based model was developed for the one-directional mass transport of species through ideal MMMs containing homogenously dispersed cubical particles. This membrane was described by the repetition of an element called RB element which contains a cubical particle in the centre and surrounded by a cube of continuous phase (Fig. 1). Therefore, the permeability of the RB element would be identical to the whole membrane in a fixed operating and manufacturing conditions. It was observed that the effective permeability of MMMs $\left(P_{E f f, R B}\right)$ is a function of the permeability in two phases $\left(P_{c}\right.$ and $P_{d}$ for continuous and dispersed phase permeabilities, respectively) as well as the volume fraction of the filler within the membrane $(\phi)$ (Eq. 1).

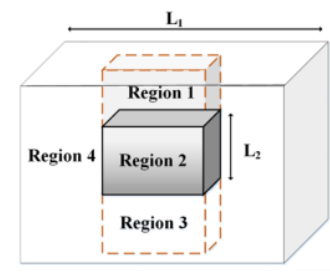

(a)

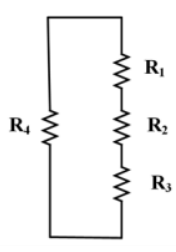

(b)

Fig 1: RB element and its equivalent resistance circuit. 


$$
P_{E f f, R B}=\left(1-\phi^{2 / 3}\right) P_{c}+\frac{P_{c} P_{d} \phi^{2 / 3}}{\phi^{1 / 3} P_{c}+\left(1-\phi^{1 / 3}\right) P_{d}}
$$

\section{FD Model}

One of the main assumptions for the RB model is the one-directional transport of the components through a mixed matrix membrane. For a neat polymeric membrane with a constant permeability or with a solid filler with identical permeability as the polymeric membrane, the concentration profile for a given component through the membrane is indeed one-directional and perfectly linear $[8,9]$. However, the presence of solid fillers within the matrix of the membrane will usually result in the deviation of the concentration profile from linear to a more tortuous diffusional pathway depending on the permeability of each phase.

As found in a separate study using numerous layers of RB elements, the effective permeability of ideal MMMs with homogenously dispersed particles is identical to its repeatable element. Therefore, the finite difference three-directional mass transfer solution was performed on one RB element to estimate the accurate effective permeability of MMMs. A zero-stage cut condition at the feed side and perfect vacuum on the permeate side of the RB element was used as the boundary conditions in the direction perpendicular to the two surfaces of the membrane. Since it is assumed that the membrane is made of a large number of identical RB elements, symmetrical boundary conditions prevail at the four faces perpendicular to the main permeation direction. Moreover, to reduce the convergence time to achieve steady state, a linear concentration profile across the thickness of the element was used as the initial condition for the numerical solution. Each direction of the RB element was discretised using 21 mesh points while the total size of the RB element was set to be 40 $\mathrm{nm}$ and the size of the central cubical particle was varied to calculate the concentration profile for different volume fractions of the dispersed phase. Mesh independency was validated for this number of mesh points. The Fick's $2^{\text {nd }}$ law of diffusion was used to obtain the steady-state concentration profile within the described RB element. Furthermore, Fick's $1^{\text {st }}$ law was used to obtain the steady state permeation flux of the species within the membrane. The effective permeability of MMMs was then calculated using the resulting permeate flux, the RB element thickness and prevailing concentration driving force.

Similar to the RB model, it was observed that the effective permeability of MMMs is a function of permeability in the two phases as well as the volume fraction of solid filler within the polymer matrix. Moreover, based on simulation results, the effective permeability of MMMs is not a function of the particle size, the membrane thickness and the feed concentration. In addition, the effect of particle shape was also investigated for cubical, cylindrical and spherical filler materials within the polymer matrix. The results demonstrate that the effect of particle shape is negligible when the volume fraction of filler within membrane is less than $40 \%$, which is much greater than the normal range of volume fraction that prevails in typical MMMs.

\section{Extended RB Model}

To develop a simple and accurate analytical model that could be used to predict rapidly the permeation flux across a mixed matrix membrane, the effective permeability for each numerical FD simulations were compared to the permeability estimated by the resistance-based model under identical conditions for the permeability of the continuous and dispersed phases as well as the volume fraction of the solid filler. This comparison allowed determining a set of correction factors $(\tau)$ for the RB model defined in terms of the ratio of the effective permeability obtained by finite differences $\left(P_{E f f, F D}\right)$ and the permeability prediction calculated with the RB model $\left(P_{E f f, R B}\right)(E q .2)$. All the individual correction factors were used to determine a global correlation that could predict the correction factor as a function of the ratio of permeabilities of the dispersed and continuous phases and the volume fraction of the dispersed phase.

$$
\tau=\frac{P_{E f f, F D}}{P_{E f f, R B}}
$$

It was observed that for a fixed filler volume fraction, a Langmuir-type equation (Eq. 3) could be used to predict the correction factor for mixed matrix membranes containing high permeable fillers (HPF) as a function of the permeability ratio of the dispersed to the continuous phase $\left(P_{d} / P_{c}\right)$. Moreover, the parameters of Langmuir-type equation $\left(b_{H P F}\right.$ and $\left.C_{M-H P F}\right)$ were found to be a function of the volumetric filler content only (Eqs. 4 and 5). 


$$
\begin{gathered}
\tau_{H P F}=1+\frac{C_{M-H P F} b_{H P F}\left(\frac{P_{d}}{P_{c}}-1\right)}{1+b_{H P F}\left(\frac{P_{d}}{P_{c}}-1\right)} \\
b_{H P F}=-0.0923 \phi^{2}-0.0563 \phi+0.1486 \\
C_{M-H P F}=2.2115 \phi^{3}-4.2545 \phi^{2}+2.043 \phi
\end{gathered}
$$

\section{Model Validation}

The validation of the extended RB model was performed using experimental data obtained in our laboratory for the pervaporation separation of butanol from aqueous binary solutions using a PDMS/Super Activated Carbon Powder membrane [10]. Fig. 2(a) present the variation of the experimental permeate fluxes of water and butanol at different operating temperatures as a function of particle volume fraction in the polymer matrix as well as the predicted values by the proposed model. A good agreement (with an average prediction error of approximately $12.5 \%$ ) was observed between the experimental and predicted values while there were some minor deviations especially for the prediction of butanol transport through the membrane.
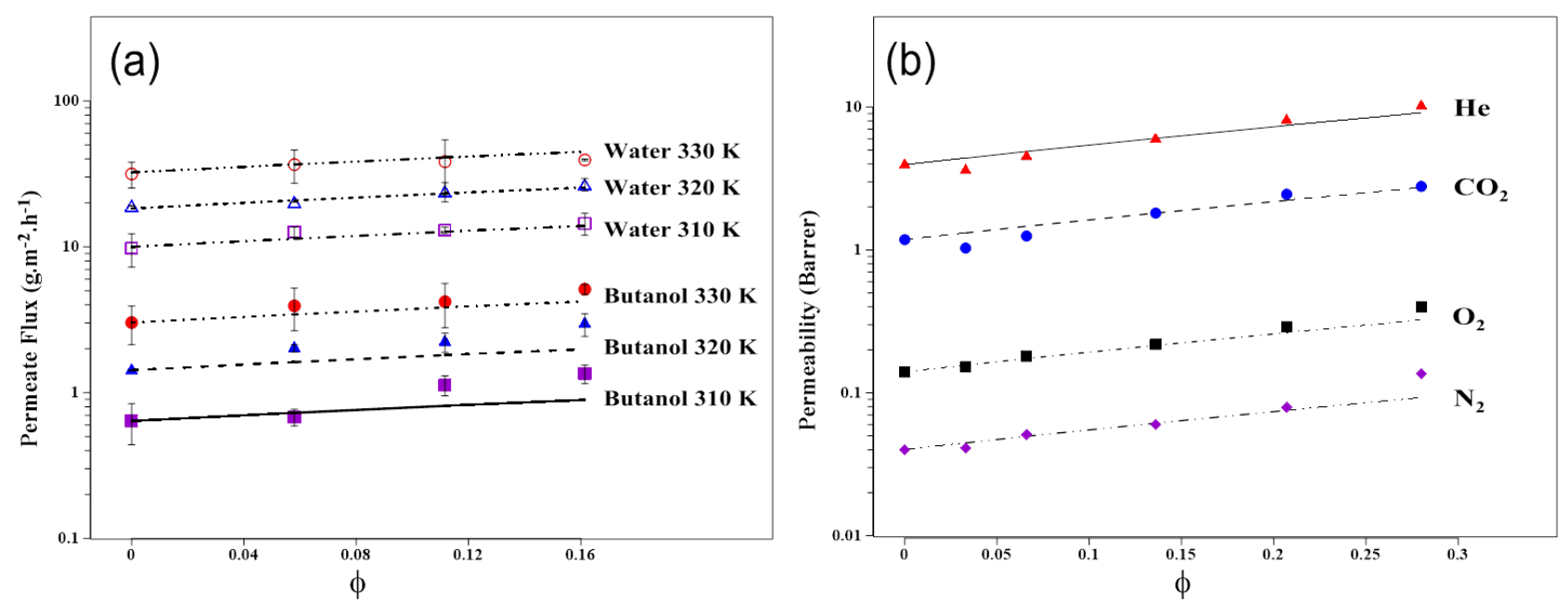

Fig. 1: Comparison between the (a) experimental [10] and predicted permeate fluxes of butanol and water as a function the volume fraction of nano activated carbon in the PDMS matrix at different operating temperatures, and (b) experimental [11] and predicted permeabilities in PVDF/MCM-41 as a function of the volume fraction of the MCM-41 in PVDF matrix.

The model validation was also performed for gas separation using experimental data obtained from the literature for the permeation of various gases through PVDF-MCM mixed matrix membranes [11]. Based on the results of Fig. 2(b) for gas separation, the model predictions show a good agreement with the experimental permeabilities of gases through this type of MMMs where the average prediction error was approximately $14.5 \%$.

\section{Conclusion}

Despite the high accuracy of the finite difference method to estimate the mass transport within the mixed matrix membranes, long computation time is an important limitation for the application of this method for various applications. Instead, in this investigation, it was desired to develop a simple resistance-based model with a correction factor based on the results of the finite difference numerical simulations to represent more accurately and more rapidly the effective permeability of mixed matrix membranes. The correction factors were modelled successfully using a Langmuir-type relation with the model parameters obtained by performing data curve fitting on the set of predicted correction factors. The correction factor is a function of the ratio of the dispersed phase permeability to the permeability of the continuous phase, and the volume fraction of the dispersed phase. The extended RB model was verified with experimental data for pervaporation and gas separation processes. High precision for the prediction of the effective permeability was observed and the model can be used with confidence to predict species permeation across mixed matrix membranes. 


\section{Acknowledgements}

The authors would like to acknowledge the financial support of the Natural Science and Engineering Research Council of Canada (NSERC).

\section{References}

[1] R. H. B. Bouma, A. Checchetti, G. Chidichimo, and E. Drioli, "Permeation through a heterogeneous membrane: the effect of the dispersed phase," J. Membr. Sci., vol. 128, no. 2, pp. 141-149, 1997.

[2] D. a. G. Bruggeman, "Berechnung verschiedener physikalischer Konstanten von heterogenen Substanzen. I. Dielektrizitätskonstanten und Leitfähigkeiten der Mischkörper aus isotropen Substanzen,” Ann. Phys., vol. 416, no. 7, pp. 636-664, 1935.

[3] L. E. Nielsen, "Models for the Permeability of Filled Polymer Systems," J. Macromol. Sci. Part - Chem., vol. 1, no. 5, pp. 929-942, 1967.

[4] L. E. Nielsen, "Thermal conductivity of particulate-filled polymers," J. Appl. Polym. Sci., vol. 17, no. 12, pp. 38193820, 1973.

[5] H. J. C. T. Hennepe, C. A. Smolders, D. Bargeman, and M. H. V. Mulder, "Exclusion and Tortuosity Effects for Alcohol/Water Separation by Zeolite-Filled PDMS Membranes," Sep. Sci. Technol., vol. 26, no. 4, pp. 585-596, 1991.

[6] R. Pal, "New Models for Thermal Conductivity of Particulate Composites," J. Reinf. Plast. Compos., vol. 26, no. 7, pp. 643-651, 2007.

[7] H. Vinh-Thang and S. Kaliaguine, "Predictive Models for Mixed-Matrix Membrane Performance: A Review," Chem. Rev., vol. 113, no. 7, pp. 4980-5028, 2013.

[8] D. M. Aguilar-Valencia, M. Á. Gómez-García, and J. Fontalvo, "Effect of pH, CO2, and High Glucose Concentrations on Polydimethylsiloxane Pervaporation Membranes for Ethanol Removal," Ind. Eng. Chem. Res., vol. 51, no. 27, pp. 9328-9334, 2012.

[9] R. Krishna and J. A. Wesselingh, "The Maxwell-Stefan approach to mass transfer," Chem. Eng. Sci., vol. 52, no. 6, pp. 861-911, 1997.

[10] H. Azimi, H. F. Tezel, and J. Thibault, "Effect of embedded activated carbon nanoparticles on the performance of Polydimethylsiloxane (PDMS) membrane for pervaporation separation of butanol," J. Chem. Technol. Biotechnol., 2017.

[11] Y. Shen and A. C. Lua, "Theoretical and experimental studies on the gas transport properties of mixed matrix membranes based on polyvinylidene fluoride," AIChE J., vol. 59, no. 12, pp. 4715-4726, 2013. 